Planetary Systems in the Universe - Observation, Formation and Evolution

Proceedings IAU Symposium No. 202, (C2004 IAU

Alan Penny, Pawel Artymowicz, Anne-Marie Lagrange, \& Sara Russell, eds.

\title{
Formation of Protoplanet Systems
}

\author{
Eiichiro Kokubo \\ National Astronomical Observatory, Mitaka, Tokyo 181-8588, Japan
}

Shigeru Ida

Tokyo Institute of Technology, Meguro-Ku, Tokyo 158-5881, Japan

\begin{abstract}
We present the latest results of large scale $(N=10000,0.5 \mathrm{AU}<$ $a<1.5 \mathrm{AU}) N$-body simulations of planetary accretion. We confirm the oligarchic growth of protoplanets in the minimum-mass disk and a more massive disk models. Protoplanets with the predicted isolation mass are formed with orbital separation of about 10-15 Hill radius.
\end{abstract}

\section{Introduction}

In the standard scenario of planetary formation, solid planets are formed through accretion of planetesimals (e.g., Safronov 1969, Hayashi et al. 1985). We have been studying planetary accretion by using $N$-body simulation. So far, we used a narrow ring of planetesimals with the surface number density of planetesimals consistent with the minimum-mass disk model (Hayashi 1981). In the present paper, we expand this model to a wide disk with a general disk model.

The wide region is necessary to see the global interaction of protoplanets and the effect of the surface number density distribution of planetesimals on planetary accretion. It is also necessary to study planetary accretion in less and more massive protoplanetary disks, which may lead to the diversity of planetary systems. The observation of protoplanetary disks revealed that the mass distribution of protoplanetary disks ranges from $1 / 10$ to 10 times the minimum-mass disk model (Sargent \& Beckwith 1996).

\section{Method of Calculation}

We perform $N$-body simulation using a 4 th-order predictor-corrector type Hermite integrator (Kokubo et al. 1998) with a special-purpose computer GRAPE-4 (Makino et al. 1997). We assume perfect accretion of bodies, in other words, any collision leads to coalesce. To shorten calculation time, we use an $f$-fold radius of bodies. The use of $f$-fold radius changes the growth timescale but not the growth mode as long as $f$ is not too large (Kokubo \& Ida 1996). For simplicity, we consider nebula gas-free cases.

The surface number density distribution of a planetesimal disk is given by the power-law distribution as $\Sigma=\Sigma_{1}(a / 1 \mathrm{AU})^{-\alpha} \mathrm{gcm}^{-2}$ and the width $0.5 \mathrm{AU} \leq$ $a \leq 1.5 \mathrm{AU}$. The number of initial bodies is $N=10000$. 

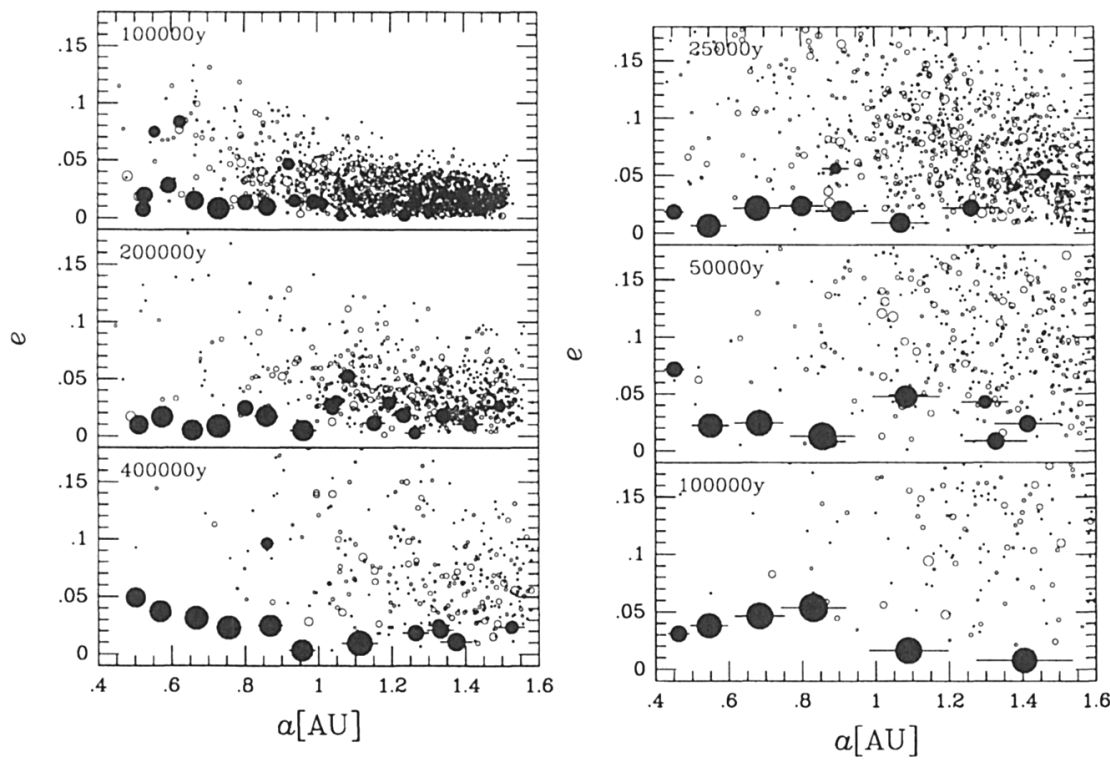

Figure 1. Evolution of planetesimal disks on the $a$-e plane for $\Sigma_{1}=10$ (left) and 100 (right) cases. The size of circles is proportional to the physical size of bodies. The length of the line on bodies is $10 r_{\mathbf{H}}$.

\section{Results}

Figures 1 and 2 show the results of the case with $\Sigma_{1}=10$ and $100, \alpha=3 / 2$, and $f=6$, where $a, e$, and $M$ denote the semimajor axis, the eccentricity, and the mass of protoplanets. The accretion propagates from small to large $a$. This is because the accretion timescale is smaller for small $a$ since the surface number density of planetesimals is higher and the orbital period is smaller for small $a$.

The growth mode of planetesimals is "runaway growth" where large planetesimals grow faster than small ones (e.g., Greenberg et al. 1978; Wetherill \& Stewart 1989). Protoplanets (runaway planetesimals) form as a result of runaway growth. The growth mode of protoplanets is called "oligarchic growth" (Kokubo \& Ida 1998, 2000). In the oligarchic growth stage, protoplanets grow in the orderly mode keeping a certain orbital separation by orbital repulsion, while most planetesimals remain small (for details, see Ida \& Kokubo in this volume).

We confirmed the oligarchic growth of protoplanets in both $\Sigma_{1}=10$ and 100 cases. Protoplanets of about the isolation mass are formed with the orbital separation $\Delta a=10-15 r_{\mathrm{H}}$, where $r_{\mathrm{H}}$ is the Hill radius of protoplanets given by $\left(M / 3 M_{\odot}\right)^{1 / 3} a$. Note that at large $a$ the mass of protoplanets is smaller than the isolation mass because accretion is still underway.

When the surface number density of planetesimals is given by $\Sigma \propto \Sigma_{1} a^{-\alpha}$, the oligarchic growth theory predicts that the isolation mass of protoplanets is $M \simeq 2 \pi a b \Sigma \propto \Sigma_{1}^{3 / 2} a^{-(3 / 2) \alpha+3}$ and their orbital separation $\Delta a \propto r_{\mathrm{H}} \propto$ $\Sigma_{1}^{1 / 2} a^{-(1 / 2) \alpha+2}$. The results of $N$-body simulations are consistent with this es- 

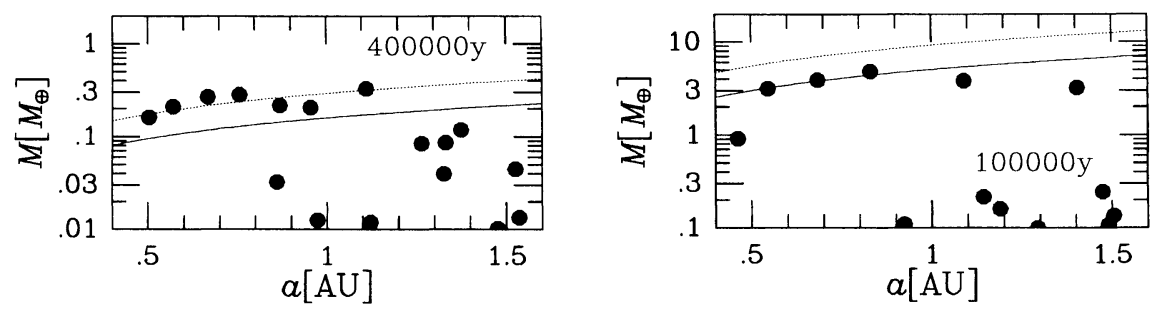

Figure 2. Snapshots of $\Sigma_{1}=10$ (right) and $\Sigma_{1}=100$ (left) planetesimal disks on the $a-M$ plane. The curves show the predicted isolation mass for $\Delta a=10 r_{\mathrm{H}}$ (solid) and $15 r_{\mathrm{H}}$ (dotted).

timates. The mass of protoplanets increases and their number decreases with $\Sigma$. Note that for the $\Sigma_{1}=100$ case, the mass of protoplanets around $1 \mathrm{AU}$ is $3-5 M_{\oplus}$, which can onset gas accretion from the nebula to become jovian planets (see Ida \& Kokubo in this volume).

\section{Summary}

We performed large scale $N$-body simulations with a general disk model and found that oligarchic growth of protoplanets is a robust result of planetary accretion. We will expand the parameter range of a planetesimal disk and systematically investigate planetary accretion from various disk models.

\section{References}

Beckwith, S. V. W., \& Sargent, A. I. 1996, Nature, 383, 139

Greenberg, R., Wacker, J., Chapman, C. R., \& Hartman, W. K. 1978, Icarus, 35, 1

Hayashi, C. 1981, Prog. Theor. Phys. Suppl., 70, 35

Hayashi, C., Nakazawa, K., \& Nakagawa, Y. 1985, in Protostars and Planets II, ed. D. C. Black \& M. S. Matthews (Tucson: Univ. of Arizona Press), 1100

Kokubo, E., \& Ida, S. 1996, Icarus, 123, 180; 1998, Icarus, 131, 171; 2000, Icarus, 143, 15

Kokubo, E., Yoshinaga, K., \& Makino, J. 1998, MNRAS, 297, 1067

Makino, J., Taiji, M., Ebisuzaki, T., \& Sugimoto, D. 1998, ApJ, 480, 432

Safronov, V. 1969, Evolution of the protoplanetary cloud and formation of the earth and planets (Moscow: Nauka Press)

Wetherill, G. W., \& Stewart, G. R. 1989, Icarus, 77, 330 